\title{
Lipoprotein(a) Is the Best Single Marker in Assessing Unstable Angina Pectoris
}

\author{
Vidosava B. Djordjević, ${ }^{1}$ Vladan Ćosić, ${ }^{2}$ Ivana Stojanović, ${ }^{1}$ Slavica Kundalić, ${ }^{2}$ \\ Lilika Zvezdanović, ${ }^{2}$ Marina Deljanin-Ilić, ${ }^{3}$ Predrag Vlahović, ${ }^{2}$ and Lidija Popović ${ }^{1}$ \\ ${ }^{1}$ Institute of Biochemistry, Faculty of Medicine, Nǐ̌, Serbia \\ ${ }^{2}$ Centre for Medical Biochemistry, Clinical Center, Niš, Serbia \\ ${ }^{3}$ Institute for Cardiovascular and Rheumatic Diseases, Niška Banja, Serbia
}

Correspondence should be addressed to Predrag Vlahović, predrag_vlahovic@yahoo.com

Received 3 September 2010; Revised 30 November 2010; Accepted 30 December 2010

Academic Editor: Ken Ichi Aihara

Copyright ( 2011 Vidosava B. Djordjević et al. This is an open access article distributed under the Creative Commons Attribution License, which permits unrestricted use, distribution, and reproduction in any medium, provided the original work is properly cited.

\begin{abstract}
This study evaluated whether statin therapy changed a diagnostic validity of lipid and inflammatory markers in ischemic heart disease (IHD) patients. Levels of lipids, lipoproteins, apolipoproteins, inflammatory markers, and atherogenic indexes were determined in 49 apparently healthy men and women, 82 patients having stable angina pectoris (SAP), 80 patients with unstable angina (USAP), and 106 patients with acute ST-elevation myocardial infarction (STEMI) treated or not treated with statins. Diagnostic accuracy of markers was determined by ROC curve analysis. Significantly lower apoA-I in all statin-treated groups and significantly higher apoB in statin-treated STEMI group compared to non-statin-treated groups were observed. CRP showed the best ROC characteristics in the assessment of STEMI patients. Lp(a) is better in the evaluation of SAP and USAP patients, considering that $\mathrm{Lp}(\mathrm{a})$ showed the highest area under the curve (AUC). Regarding atherogenic indexes, the highest AUC in SAP group was obtained for TG/apoB and in USAP and STEMI patients for TG/HDL-c. Statins lowered total cholesterol, LDL-c, and TG but fail to normalize apoA-I in patients with IHD.
\end{abstract}

\section{Introduction}

Beside endothelial dysfunction leading to inflammatory reaction, lipid metabolism disorders represent the second key event in the initiation and rapid development of atherogenesis [1]. Many individual lipid and inflammatory markers have been considered as the factors playing an important role in atherogenesis and prognosis of related diseases. The atherogenic dyslipidemic profile, especially mild to marked elevation of apo-B containing lipoproteins, such as very low-density lipoproteins (VLDL), VLDL-remnants, intermediate-density lipoproteins (IDL), and low-density lipoproteins (LDL) (specifically small, dense LDL), and low levels of high-density lipoproteins (HDL) [2-4], appears to promote enhanced arterial cholesterol deposition and accelerate the progression of atherosclerotic disease. Despite the use of new and effective pharmacological drugs to lower plasma lipid concentration, cardiovascular diseases continue to be the main cause of death in western countries [5, 6]. Fenofibrate lowers the plasma level of cholesterol and triglyceride, corrects the abnormality in LDL metabolism, but has no effect on HDL-cholesterol (HDL-c) [7]. On the other hand, statins have a therapeutic effect on lipid metabolism and inflammation. They lower total and LDLcholesterol (LDL-c), elevate HDL-c, and lower inflammatory markers such as C-reactive protein (CRP) [8-11]. Since antilipidemic drugs induce modifications in current lipid metabolism and inflammatory response, lipid and inflammatory markers become less convenient in assessing the activity of atherosclerotic process. This may be a reason for contradictory results related to CRP, which was until recently a promising marker in predicting cardiovascular events. Recent limited data have shown that lipoprotein(a) $(\operatorname{Lp}(\mathrm{a}))$ and the ratio of Tc/HDL-c may be used as much stronger 
predictors in screening for high blood lipid [12], and Lp(a) is the best single marker for the presence of cerebrovascular disease [13].

The aim of this study was to determine which individual lipid or inflammatory biomarkers had the highest clinical accuracy by Receiver Operating Characteristic (ROC) curve analysis in patients with different stages of ischemic heart disease treated or not treated with statins.

\section{Materials and Methods}

2.1. Subject. The study evaluated patients admitted to the Institute for Cardiovascular diseases "Niška Banja" for the evaluation of chest pain. Patients were categorized into three groups based on the degree of ischemic heart disease (IHD): chronic stable angina pectoris (SAP group), unstable angina pectoris (USAP group), and acute ST-elevation myocardial infarction (STEMI group). The SAP group consisted of 52 male and 30 female (total 82 , mean age $61.3 \pm 6.5$ years); the USAP group had 50 male and 30 female (total 80 mean age $60.8 \pm 9.8$ years); the STEMI patients included 72 male and 34 female (total 106, mean age $60.22 \pm 12.7$ years). Each group was divided into statin-treated $(+)$ and non-statintreated $(-)$ groups. The $\mathrm{SAP}(+)$ group consisted of 34 males and 17 females, the $\operatorname{SAP}(-)$ of 18 males and 13 females, the $\operatorname{USAP}(+)$ of 35 males and 12 females, the $\operatorname{USAP}(-)$ of 15 males and 18 females, the STEMI $(+)$ of 37 males and 18 females, and the STEMI $(-)$ of 35 males and 16 females.

The patients in the SAP group gave a history consistent with stable angina for at least 3 months before entering the study and demonstrated objective evidence of ischemia on exercise electrocardiogram and/or stress echocardiogram. None of the patients in this group had previous myocardial infarction or myocardial revascularization, cardiac valve disease, cardiomyopathy, malignant arrhythmias, acute or chronic liver disease, renal failure, or inflammatory disease, and none of the patients were under consideration for coronary revascularization at the time of the inclusion into the study.

Unstable angina was defined according to Hamm and Braunwald [14]. All patients in the USAP group had chest pain of increasing frequency and severity or at rest during the last 48 hours before hospitalisation associated with ST segment changes, $\mathrm{T}$ wave changes, or both and without rise in cardiac enzymes and troponin I.

Acute myocardial infarction was based on the following criteria: chest pain persisting longer than $30 \mathrm{~min}$, concomitant changes on the electrocardiogram at the admission to hospital and elevated troponin I levels. All patients had STEMI according to the Guidelines of the European Society of Cardiology [15]. In all patients, a detailed clinical analysis was performed just after admission, and all of them were asked for current medications. Additional explanation of the criteria for patients with ischemic heart disease selection was given in our previously published paper [16].

For the control group, we recruited 49 (30 male, 19 female) healthy volunteers-blood bank donors from the Department for Blood Transfusion of the Clinical Centre Niš.
All controls were free of any acute infectious disease and any history of hypertension, diabetes or ischemic heart disease. All subjects gave informed consent prior to their enrolment in the study, and the study was approved by the local Ethics Committee.

2.2. Methods. Blood samples were obtained within 24 hours after admission after overnight fasting. Peripheral venous blood was drown into vacutainer tubes containing ethylene diamine-tetracetic acid (EDTA), citrate, or no anticoagulant. Troponin I and inflammatory markers were determined in serum, fibrinogen in citrate plasma, and lipid markers in EDTA plasma. Aliquots of plasma and serum for the determination of neopterin, iNOS, $\mathrm{NO}_{2} / \mathrm{NO}_{3}, \mathrm{TNF}-\alpha, \mathrm{Lp}(\mathrm{a})$, and oxidized LDL (oxLDL) were stored at $80^{\circ} \mathrm{C}$ until assayed. All other analyses were performed the same day the blood was collected.

Troponin I was determined on AxSYM (Abbott Ireland Diagnostics Division, Lisnamuck, Ireland). The diagnostic cutoff for acute myocardial infarction is $0.40 \mathrm{ng} / \mathrm{mL}$. In apparently healthy population, 99th percentile is $0.04 \mathrm{ng} / \mathrm{mL}$. Depending on patient hours after admission, sensitivity ranges from $60 \%(0-6 \mathrm{~h})$ to $91.7 \%(12-24 \mathrm{~h})$, and specificity $97.4 \%$ to $98.3 \%$.

HsCRP, total cholesterol (Tc), triglycerides (TG), LDLc, HDL-c, apolipoprotein A-I (ApoA-I), apolipoprotein $\mathrm{B}$ (ApoB) were assayed on analyzer AU 400 (Olympus, Tokio, Japan). HsCRP was measured using a latex-enhanced immunoturbidimetric method on Olympus AU400. hsCRP reference values are $<1 \mathrm{mg} / \mathrm{L}$ (manufacturer recommendation). This test is linear within a concentration range of $0.08-$ $80 \mathrm{mg} / \mathrm{L}$. The intra-assay precision for three different samples are between CV\% 0.55 and CV\% 4.32. Tc, TG, HDL-c, and LDL-c were measured by routine methods on analyzer AU 400. ApoA-I and apoB concentrations were determined using an immunoturbidimetric method (Olympus, Tokio, Japan).

The plasma concentration of $\operatorname{Lp}(\mathrm{a})$ was also assayed by a latex immunoassay (Sentinel CH Srl Diagnostics, Milan, Italy) on Olympus AU400. Lower detection limit was $3 \mathrm{mg} / \mathrm{dL}$ and the reference values were $<30 \mathrm{mg} / \mathrm{dL}$. The performance characteristics of $\mathrm{Lp}(\mathrm{a})$ test were as follows: the measuring range was $3.0-130 \mathrm{mg} / \mathrm{dL}$; the intra-assay precision determined in two levels was CV\% 2.00 and 1.26, respectively.

Serum neopterin concentrations were determined using a commercially available immunoassay (ELISA kit, IBL, Hamburg, Germany). The limit of detection was $0.7 \mathrm{nmol} / \mathrm{L}$.

Serum iNOS activity was measured by a commercially available Quantikine human iNOS immunoassay (ELISA kit, R\&D Systems Europe, LTD. UK). The limit of detection was $0.15 \mathrm{U} / \mathrm{mL}$.

Quantikine human TNF- $\alpha$ immunoassay (R\&D Systems Europe, Ltd. UK) was used for the estimation of serum TNF$\alpha$ concentration.

Serum $\mathrm{NO}_{2} / \mathrm{NO}_{3}$ concentration was measured by the modified cadmium-reduction method of Navaro-Gonzálvez et al. [17] which is based on the produced nitrite determination by diazotization of sulfanilamide and coupling to naphthylene diamine. The lower and upper detection 
TABle 1: Demographic characteristics and blood lipid and inflammatory markers in patients with ischemic heart disease.

\begin{tabular}{|c|c|c|c|c|}
\hline & SAP & USAP & STEMI & Controls \\
\hline Male/female $(N)$ & $52 / 30$ & $50 / 30$ & $72 / 34$ & $30 / 19$ \\
\hline Age years & $61.3 \pm 6.5$ & $60.8 \pm 9.8$ & $60.2 \pm 12.7$ & $59.1 \pm 9.7$ \\
\hline Troponin I, ng/mL & $0.0(0.0-1.68)$ & $0.03(0.0-3.7)^{*}$ & $12.8(0.0-159.6)^{* * *}$ & $0.0(0.0-0.01)$ \\
\hline $\mathrm{TG}, \mathrm{mmol} / \mathrm{L}$ & $1.88(0.58-21.4)^{*}$ & $2.08(0.56-7.6)^{* * *}$ & $1.90(0.56-4.9)^{*}$ & $1.30(0.46-4.18)$ \\
\hline $\mathrm{Tc}, \mathrm{mmol} / \mathrm{L}$ & $5.60 \pm 1.20$ & $5.78 \pm 1.72$ & $5.91 \pm 1.57$ & $5.48 \pm 0.49$ \\
\hline $\mathrm{HDL}-\mathrm{c}, \mathrm{mmol} / \mathrm{L}$ & $1.16 \pm 0.28$ & $1.00 \pm 0.31^{* * *}$ & $1.06 \pm 0.26^{* * *}$ & $1.15 \pm 0.28$ \\
\hline LDL-c, mmol/L & $3.47 \pm 1.05$ & $3.55 \pm 1.40$ & $3.88 \pm 1.28$ & $3.51 \pm 0.59$ \\
\hline $\mathrm{Lp}(\mathrm{a}), \mathrm{mg} / \mathrm{dL}$ & $14.4(3.6-67.8)^{* * *}$ & $22.7(5.9-88.9)^{* * *}$ & $17.8(4.8-79.0)^{* * *}$ & $5.6(2.9-19.7)$ \\
\hline ApoA-I, g/L & $1.13 \pm 0.30^{* * *}$ & $1.04 \pm 0.29^{* * *}$ & $1.11 \pm 0.17^{* * *}$ & $1.34 \pm 0.21$ \\
\hline ApoB, g/L & $1.06 \pm 0.38$ & $1.17 \pm 0.40$ & $1.25 \pm 0.31$ & $1.17 \pm 0.14$ \\
\hline oxLDL, ng/mL & $211(11.7-1925)$ & $143(7-1550)^{*}$ & $140(15-2105)$ & $195(18-705)$ \\
\hline hsCRP, g/L & $3.0(0.4-84.35)^{*}$ & $5.8(0.43-178.3)^{* *, \mathrm{~b}}$ & $18.0(0.56-270)^{* * *, \mathrm{c}}$ & $1.5(0.1-16.5)$ \\
\hline Fibrinogen, g/L & $4.29 \pm 1.22$ & $5.03 \pm 1.49^{* *}$ & $5.06 \pm 2.46^{*}$ & $3.92 \pm 1.03$ \\
\hline Neopterin, nmol/L & $14.9(3-57)^{* * *}$ & $14.3(1-105)^{* *, \mathrm{a}}$ & $13.0(1-52)$ & $13.4(1-19.6)$ \\
\hline $\mathrm{iNOS}, \mathrm{U} / \mathrm{mL}$ & $2.2(0.5-4.7)$ & $2.9(0.9-6.0)$ & $3.0(0.9-6.2)$ & $2.2(0.6-9.3)$ \\
\hline $\mathrm{NO}_{2} / \mathrm{NO}_{3}, \mu \mathrm{mol} / \mathrm{L}$ & $96(35-197)$ & $125(52-199)^{* *, \mathrm{~b}}$ & $102(61-243)$ & $97(57-230)$ \\
\hline TNF- $\alpha, p g / m L$ & $12.74 \pm 1.25$ & $13.78 \pm 1.93$ & $14.47 \pm 2.15$ & $13.60 \pm 2.19$ \\
\hline
\end{tabular}

The results are presented as means \pm SD for parametric, and median (min-max) for nonparametric values $* P<.05$ versus controls, $* * P<.01$ versus controls, ${ }^{* * *} P<.001$ versus controls, ${ }^{\mathrm{a}} P<.05$ versus STEMI, ${ }^{\mathrm{b}} P<.01$ versus $\mathrm{SAP},{ }^{\mathrm{c}} P<.001$ versus SAP.

limits were 2 and $250 \mu \mathrm{mol} / \mathrm{L}$, respectively. Plasma oxLDL concentrations were estimated by a commercially available ELISA kit (Immune diagnostic AG, Bensheim).

2.3. Statistical Analysis. Most statistics were performed using SPSS (the Statistical Package for the Social Sciences) computer program. The comparison of the different patient groups to the control group was performed using ANOVA followed by a 2-sided Dunnett's test (for multiple comparisons), or Student's nonpaired $t$-test as appropriate. The clinical accuracy of the examined parameters was assessed using receiver operating characteristic (ROC) curve analysis. ROC plots were constructed, and the areas under the curves (AUC), standard errors, 95\% confidence interval, sensitivity, and specificity as well as optimal cutoff were calculated using MedCalc computer program. Cutoff values at which the discrimination between the cases with positive and negative diagnosis was optimal were set. The comparisons of the areas under different ROC plots were made using univariate $\mathrm{z}$ scores. Multinomial logistic regression was used to assess wich of the lipid and inflammatory markers showing high sensitivity and specificity may be the best predictor of SAP, USAP or STEMI.

\section{Results}

Demographic characteristics in the studied patients showed the prevalence of males in all groups. The average age of groups was about sixty (Table 1). Statistical analysis using ANOVA showed that SAP patients had significantly higher concentrations of TG, $\mathrm{Lp}(\mathrm{a})$, hsCRP, neopterin, and significantly lower levels of apoA-I in comparison with healthy subjects. In addition to these parameters in the USAP group, three more markers were significantly increased, including troponin $\mathrm{I}, \mathrm{NO}_{2} / \mathrm{NO}_{3}$ and fibrinogen, while HDL$c$ and oxLDL were decreased. In STEMI patients significant differences were observed in troponin I, TG, HDL-c, Lp(a), apoA-I, hsCRP and fibrinogen. Also, significant differences were found in hsCRP, neopterin and $\mathrm{NO}_{2} / \mathrm{NO}_{3}$ between different patient groups (Table 1).

However, the testing between statin-treated and nonstatin-treated groups showed fewer differences. None of all studied inflammatory markers showed any significant difference between statin-treated and non-statin-treated groups (Table 2). Among lipid markers only apoA-I levels were significantly decreased in $\mathrm{SAP}(+)$ compared to $\mathrm{SAP}(-)$ patients. A significant decrease in apoA-I, HDL-c, and LDLc/apoB was noted in USAP $(+)$ patients, as well as a significant increase in apoB/apoA-I, LDL-c/HDL-c, and Tc/HDL$c$, in comparison with the $\operatorname{USAP}(-)$ group. STEMI $(+)$ patients showed a significant decrease in apoA-I and LDL-c/apoB and an increase in apoB, apoB/apoA-I, LDLc/HDL-c and Tc/HDL-c compared to STEMI $(-)$ patients (Table 3).

Since we did not find any significant difference in ROC curve analysis between statin-treated and non-statin-treated groups in any individual inflammatory or lipid marker, ROC curve analysis included both subgroups of each patient group.

The ROC curves for inflammatory markers are presented in Figure 2. In SAP patients no biomarker showed a significant difference related to any other although hsCRP had the highest area under the ROC $(0.691 \pm 0.079)$ (Table 4). The greatest sensitivity was found for $\mathrm{NO}_{2} / \mathrm{NO}_{3}(81.0 \%)$, TNF- $\alpha$ (88.0\%), and neopterin (88.6\%), but their specificities were low $(30.4 \%, 42.1 \%$, and $42.9 \%$, resp.). 


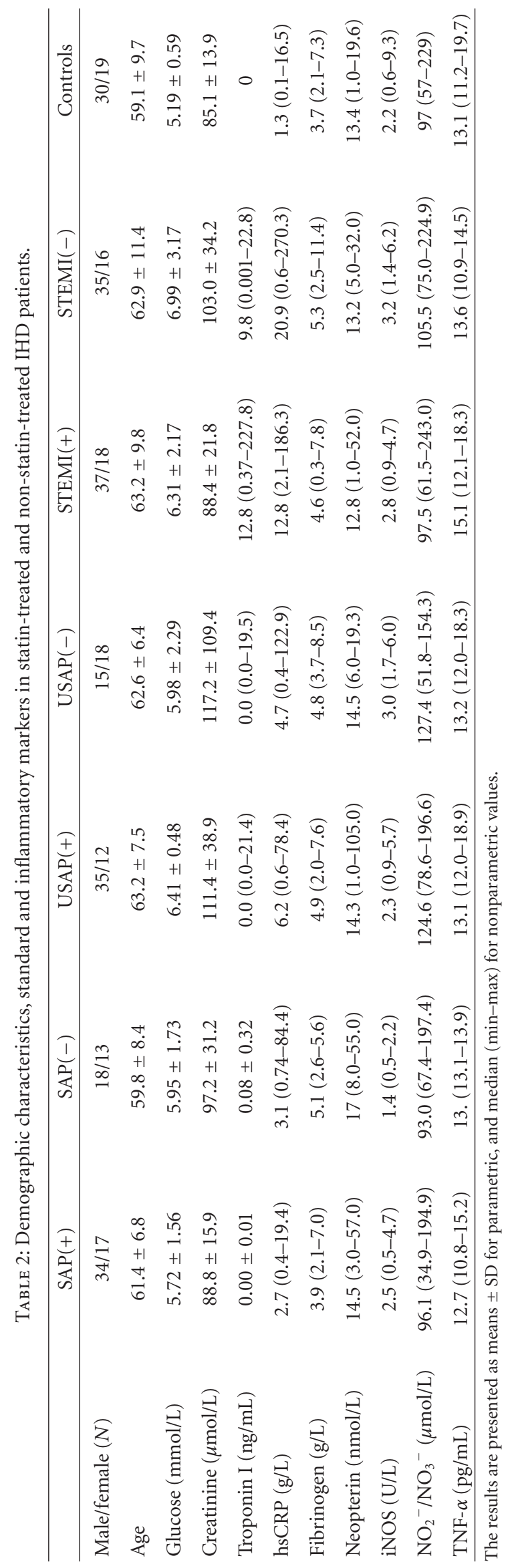




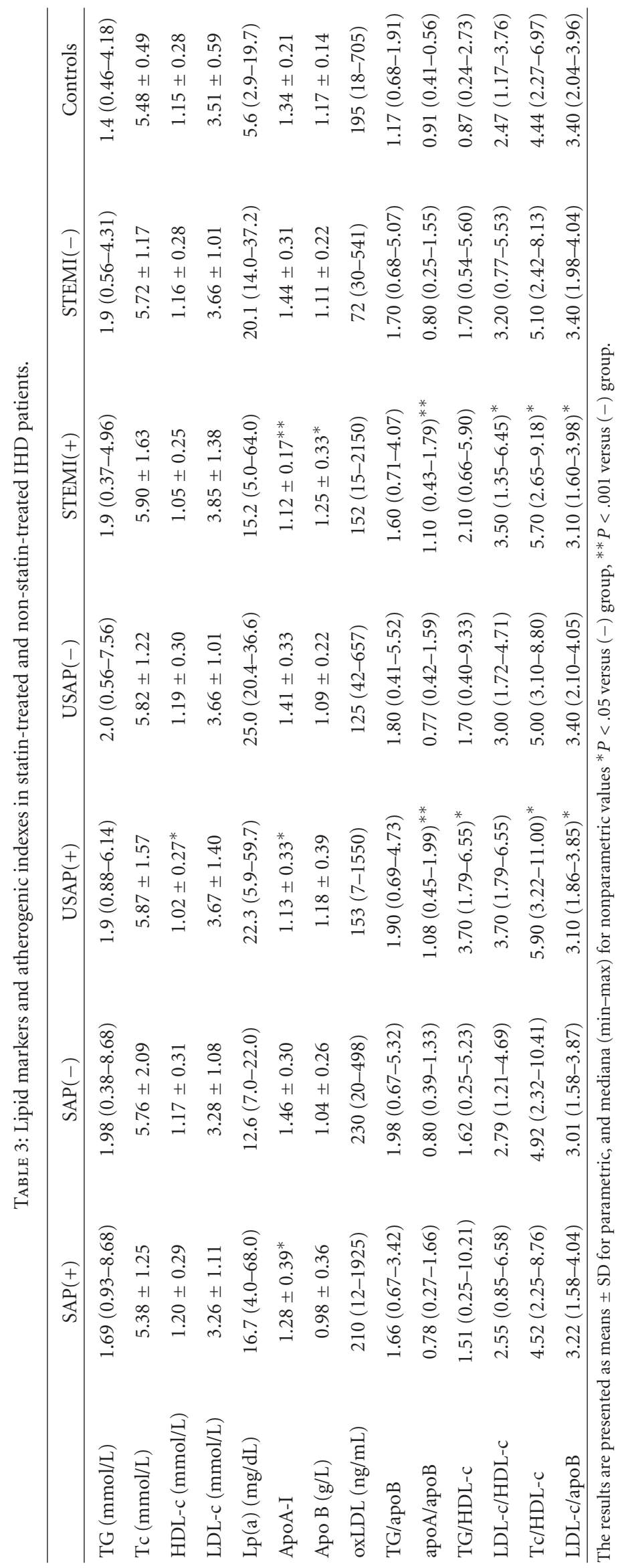


TABLE 4: The results of ROC curves analysis of inflammatory markers in ischemic heart disease patients.

\begin{tabular}{|c|c|c|c|c|c|c|c|}
\hline & & AUC & SE & 95\%CI & Specificity & Sensitivity & Criterion \\
\hline \multirow{6}{*}{ SAP } & CRP & 0.691 & 0.079 & $0.533-0.821$ & 66.7 & 72.7 & $>1.9$ \\
\hline & Fibrinogen & 0.557 & 0.087 & $0.399-0.706$ & 59.5 & 75.9 & $>3.9$ \\
\hline & iNOS & 0.558 & 0.087 & $0.400-0.707$ & 78.9 & 48.0 & $>2.8$ \\
\hline & Neopterin & 0.642 & 0.086 & $0.484-0.781$ & 42.9 & 88.6 & $>11.9$ \\
\hline & $\mathrm{NO}_{2} / \mathrm{NO}_{3}$ & 0.567 & 0.087 & $0.410-0.716$ & 30.4 & 81.0 & $\leq 122.0$ \\
\hline & TNF- $\alpha$ & 0.596 & 0.088 & $0.437-0.741$ & 42.1 & 88.0 & $\leq 14.0$ \\
\hline \multirow{6}{*}{ USAP } & CRP & $0.808^{*}$ & 0.074 & $0.643-0.919$ & 87.2 & 69.8 & $>4.1$ \\
\hline & Fibrinogen & 0.680 & 0.090 & $0.504-0.825$ & 59.5 & 90.5 & $>3.9$ \\
\hline & iNOS & 0.610 & 0.095 & $0.433-0.767$ & 78.9 & 52.6 & $>2.8$ \\
\hline & Neopterin & 0.709 & 0.086 & $0.534-0.848$ & 94.3 & 34.9 & $>18.9$ \\
\hline & $\mathrm{NO}_{2} / \mathrm{NO}_{3}$ & 0.698 & 0.088 & $0.523-0.839$ & 47.8 & 92.7 & $>88.6$ \\
\hline & TNF- $\alpha$ & 0.548 & 0.097 & $0.374-0.714$ & 31.6 & 94.7 & $>11.9$ \\
\hline \multirow{6}{*}{ STEMI } & CRP & $0.923^{* *}$ & 0.043 & $0.796-0.982$ & 89.7 & 88.6 & $>4.7$ \\
\hline & Fibrinogen & 0.562 & 0.090 & $0.399-0.716$ & 91.9 & 52.2 & $>5.0$ \\
\hline & iNOS & 0.602 & 0.088 & $0.437-0.751$ & 21.1 & 100.0 & $>0.9$ \\
\hline & Neopterin & 0.725 & 0.081 & $0.563-0.852$ & 100.0 & 19.0 & $>19.6$ \\
\hline & $\mathrm{NO}_{2} / \mathrm{NO}_{3}$ & 0.627 & 0.087 & $0.462-0.772$ & 47.8 & 74.4 & $>88.6$ \\
\hline & TNF- $\alpha$ & 0.604 & 0.088 & $0.439-0.753$ & 89.5 & 27.3 & $>15.5$ \\
\hline
\end{tabular}

${ }^{*} P<.05$ versus TNF- $\alpha,{ }^{* *} P<.05$ to $P<.001$ versus all other markers.

Also, in USAP patients AUC for hsCRP was the highest $(0.808 \pm 0.074)$, and significantly higher only in comparison with TNF- $\alpha(0.548 \pm 0.097)$. Fibrinogen, $\mathrm{NO}_{2} / \mathrm{NO}_{3}$, and TNF- $\alpha$ showed very high sensitivity $(90.5 \%, 92.7 \%$, and $94.7 \%$, resp.) for optimal cutoff values, and low specificity (under 59.5\%). Neopterin showed high specificity (94.3\%), and rather poor sensitivity (34.9\%). The best predictor for disease activity was CRP with specificity of $87.2 \%$, and sensitivity $69.8 \%$ when the cutoff was $>4.1 \mathrm{mg} / \mathrm{L}$.

In the STEMI group, ROC for hsCRP $(0.923 \pm 0.043)$ was significantly higher in comparison with all others. The highest sensitivity was obtained for iNOS (100\%), but very low specificity $(21.1 \%)$ excluded this marker from clinical use. Due to good sensitivity (88.6\%) and specificity (89.7\%) for cutoff $>4.7$, CRP alone may be satisfactory for patient screening.

The results related to ROC curves of lipid markers were shown in Figure 1 and Table 5. The highest AUC in SAP patients was obtained for $\operatorname{Lp}(a)(0.835 \pm 0.049)$, which is significantly higher than AUC for oxLDL, LDL-c and apoB $(P<.001)$. ApoA-I also had the AUC $(0.780 \pm$ $0.065)$ significantly higher than AUC for oxLDL and LDLc $(P<.05)$. However, sensitivity $(81.8 \%)$, and specificity $(80.0 \%)$ of $\operatorname{Lp}(\mathrm{a})$ were higher compared to all the other lipid markers.

In patients with USAP the highest AUCs were observed for $\operatorname{Lp}(\mathrm{a})(0.951 \pm 0.025$, significantly higher in comparison with LDL-c, oxLDL, apoB and TG), and apoA-I (0.876 \pm 0.051 , significantly higher than apoB and oxLDL AUCs). Sensitivity and specificity for $\operatorname{Lp}(\mathrm{a})$ were very high $(97.9 \%$ and $84.0 \%$, resp.), while for apoA-I these values were $91.3 \%$ and $71.8 \%$, respectively. So, these markers may be useful in assessing disease activity in more than $90 \%$ of patients.
No difference between the ability of $\operatorname{Lp}(\mathrm{a})$ and apoA-I to correctly stratify the patients was found.

Further, in STEMI patients both $\operatorname{Lp}(\mathrm{a})$ and apoA-I had also the highest AUC in comparison with other studied markers. For Lp(a) AUC was 0.881 and statistically different in comparison with apoB, LDL-c, oxLDL $(P<.001)$ and TG $(P<.01)$, and for apoA-I it was 0.836 (significantly higher than AUCs for LDL-c and oxLDL; $P<.001)$. OxLDL, apoB and LDL-c showed high specificity $(96.7 \%, 89.7 \%$ and $87.2 \%$, resp.), but very poor sensitivity $(24.5 \%, 30.9 \%$, and $25.5 \%$, resp.), which made them unsatisfactory for diagnostic screening. Thus, $\mathrm{Lp}(\mathrm{a})$ with $84.9 \%$ sensitivity and $84.0 \%$ specificity stayed the best predictor, better than apoA-I whose sensitivity was $83.6 \%$ and specificity $71.8 \%$.

Since hsCRP showed the best characteristics among all studied inflammatory markers, and $\operatorname{Lp}(\mathrm{a})$ was the best lipid marker, we compared these two and troponin I in patient groups and showed that (Table 6) there was a significant difference between their AUCs in USAP patients in whom AUC for $\mathrm{Lp}(\mathrm{a})$ was $0.957(P<.05)$ in comparison with 0.859 for hsCRP AUC and 0.821 for troponin I AUC $(P=.015)$. In SAP patients, there was no significant difference in AUC between the different markers although hsCRP AUC and Lp(a) AUC were significantly higher $((P<.05), P<.01$, resp.) than AUC for troponin I.

Generally, almost all atherogenic indexes showed acceptable discriminative ability for ischemic heart disease patients (Figure 3, Table 7). The ratio of TG to apoB had the highest AUC $(0.89 \pm 0.03)$ and significantly higher than AUCs for apoB/apoA $(0.71 \pm 0.06)$, LDL-c/apoB $(0.77 \pm 0.06)$, LDLc/HDL-c $(0.65 \pm 0.06)$, and Tc/HDL-c $(0.73 \pm 0.06)$ in SAP patients. A similar relationship was observed for TG/HDLc with AUC of $0.88 \pm 0.04$. Identical AUCs were found 
TABLE 5: The results of ROC curves analysis of lipid markers in ischemic heart disease patients.

\begin{tabular}{|c|c|c|c|c|c|c|c|}
\hline & & AUC & SE & $95 \% \mathrm{CI}$ & Specificity & Sensitivity & Criterion \\
\hline \multirow{6}{*}{ SAP } & ApoA-I & $0.780^{\mathrm{a}}$ & 0.065 & $0.660-0.873$ & 71.8 & 77.3 & $\leq 1.28$ \\
\hline & ApoB & $0.542^{\mathrm{b}}$ & 0.076 & $0.414-0.666$ & 92.3 & 38.6 & $\leq 0.9$ \\
\hline & LDL-c & $0.539^{\mathrm{b}}$ & 0.076 & $0.411-0.664$ & 87.2 & 40.9 & $\leq 3.06$ \\
\hline & $\mathrm{Lp}(\mathrm{a})$ & $0.835^{c}$ & 0.049 & $0.722-0.915$ & 80.0 & 81.8 & $>8.2$ \\
\hline & oxLDL & 0.552 & 0.075 & $0.423-0.675$ & 80.0 & 43.2 & $>280$ \\
\hline & TG & 0.704 & 0.065 & $0.578-0.811$ & 48.7 & 86.4 & $>1.31$ \\
\hline \multirow{6}{*}{ USAP } & ApoA-I & $0.876^{\mathrm{C}}$ & 0.051 & $0.773-0.944$ & 71.8 & 91.3 & $\leq 1.28$ \\
\hline & ApoB & 0.557 & 0.074 & $0.430-0.678$ & 89.7 & 21.7 & $>1.41$ \\
\hline & LDL-c & 0.588 & 0.075 & $0.461-0.707$ & 64.1 & 60.9 & $\leq 3.49$ \\
\hline & $\mathrm{Lp}(\mathrm{a})$ & $0.951^{\mathrm{B}}$ & 0.025 & $0.869-0.988$ & 84.0 & 97.9 & $>8.2$ \\
\hline & oxLDL & 0.529 & 0.075 & $0.403-0.652$ & 93.3 & 32.6 & $>460$ \\
\hline & TG & $0.742^{\mathrm{A}}$ & 0.060 & $0.621-0.841$ & 71.8 & 69.6 & $>1.94$ \\
\hline \multirow{6}{*}{ STEMI } & ApoA-I & $0.836^{* * * *}$ & 0.057 & $0.732-0.911$ & 71.8 & 83.6 & $\leq 1.26$ \\
\hline & ApoB & $0.626^{* *}$ & 0.068 & $0.506-0.735$ & 89.7 & 30.9 & $>1.41$ \\
\hline & LDL-c & 0.514 & 0.073 & $0.395-0.631$ & 87.2 & 25.5 & $\leq 3.07$ \\
\hline & $\mathrm{Lp}(\mathrm{a})$ & $0.881^{* * * / * * * * *}$ & 0.038 & $0.785-0.994$ & 84.0 & 84.9 & $>8.2$ \\
\hline & oxLDL & 0.505 & 0.074 & $0.387-0.623$ & 96.7 & 24.5 & $>4.80$ \\
\hline & TG & $0.664 *$ & 0.065 & $0.546-0.769$ & 56.4 & 69.1 & $>1.49$ \\
\hline
\end{tabular}

${ }^{\mathrm{a}} P<.05$ versus LDL-c and oxLDL, ${ }^{\mathrm{b}} P<.05$ versus TG, ${ }^{\mathrm{c}} P<.001$ versus oxLDL, LDL-c and ApoB, ${ }^{\mathrm{A}} P<.05$ versus ApoB and OxLDL, ${ }^{\mathrm{B}} P<.001$ versus LDL-c, oxLDL, ApoB and TG, ${ }^{C} P<.001$ versus ApoB, LDL-c and oxLDL, ${ }^{*} P<.05$ versus ApoA, LDL-c, ${ }^{* *} P<0.01$ versus ApoA, LDL-c, ${ }^{* * *} P<.01$ versus TG, $* * * * P<.001$ versus LDL-c, oxLDL, $* * * * * P<.001$ versus ApoB, LDL-c, OxLDL.

TABLE 6: Comparison of CRP and Lp(a) and troponin I ROC curves analysis.

\begin{tabular}{|c|c|c|c|c|c|c|c|}
\hline & & AUC & SE & $95 \% \mathrm{CI}$ & Specificity & Sensitivity & Cutoff \\
\hline \multirow{3}{*}{ SAP } & CRP & $0.758^{* *}$ & 0.057 & $0.640-0.853$ & 57.6 & 75.7 & $>1.9$ \\
\hline & $\operatorname{Lp}(\mathrm{a})$ & $0.843^{* * *}$ & 0.046 & $0.736-0.919$ & 80.0 & 81.8 & $>8.2$ \\
\hline & Troponin I & 0.568 & 0.049 & $0.480-0.654$ & 96.9 & 17.6 & $>0$ \\
\hline \multirow{3}{*}{ USAP } & CRP & 0.859 & 0.043 & $0.750-0.930$ & 87.2 & 69.8 & $>4.1$ \\
\hline & $\operatorname{Lp}(\mathrm{a})$ & $0.957^{*}$ & 0.023 & $0.880-0.990$ & 84.0 & 97.9 & $>8.2$ \\
\hline & Troponin I & 0.821 & 0.038 & $0.743-0.883$ & 96.8 & 63.5 & $>0$ \\
\hline \multirow{3}{*}{ STEMI } & CRP & 0.946 & 0.024 & $0.870-0.984$ & 89.7 & 88.6 & $>4.7$ \\
\hline & $\operatorname{Lp}(\mathrm{a})$ & 0.888 & 0.036 & $0.796-0.948$ & 84.0 & 84.9 & $>8.2$ \\
\hline & Troponin I & 1.000 & 0.000 & $0.971-1.000$ & 100.0 & 93.4 & $>0.01$ \\
\hline
\end{tabular}

${ }^{*} P<.05$ versus CRP and troponin I in USAP patients, ${ }^{* *} P<.05$ versus troponin I in SAP patients, ${ }^{* * *} P<0.001$ versus troponin I in SAP patients.

for apoB/apoA and TG/HDL-c ratios $(0.89 \pm 0.03)$ and a little bit lower AUC for Tc/HDL-c $(0.87 \pm 0.03)$ in USAP patients. All three AUCs were significantly higher than AUC for LDL-c/HDL-c. The highest sensitivity and specificity were observed for TG/HDL-c $(80.0 \%$ and $82.4 \%$, resp.), and for Tc/HDL-c $(73.3 \%$ and $71.4 \%$, resp.). In STEMI patients AUC for TG/HDL-c was the highest $(0.91 \pm 0.03)$, followed by Tc/HDL-c $(0.89 \pm 0.03)$, and apoB/apoA (0.87 $\pm 0.04)$. Satisfactory sensitivity and specificity were noted for TG/apoB (78.6\% and 74.3\%, resp.).

Multinomial logistic regression was used to adequately predict $(H=203.12, d f=15, P<.0005)$ a classification of cases into evaluated groups. This model classified evaluated cases into the SAP group with an accuracy of $87.9 \%$, control group $82.6 \%$, STEMI group with $78.4 \%$, and USAP group with 57.6\%. Pearson $(H=337.53, d f=402, P=.99)$ and
Deviance $(H=173.73, d f=402, P=1)$ confirmed that this model adequately fited the data. Obtained model explained about $82 \%$ of variation in evaluated groups. Likelihood ratio test showed that $\mathrm{Lp}(\mathrm{a})$, apoA-I, TG, and CRP were predictors significantly $(P<.0005)$ contributing to the obtained model. In comparison to the control group, $\mathrm{Lp}(\mathrm{a})(\mathrm{OR}=1.27,95 \%$ CI 1.11 to $1.45, P=.001)$ and apoA-I $(\mathrm{OR}=8.295$, CI $95 \% 1.22$ to $56.35, P=.03$ ) represented significant, while TG $(\mathrm{OR}=0.23,95 \%$ CI 0.04 to $1.52, P=0.13)$, CRP $(\mathrm{OR}=1.36,95 \%$ CI 0.97 to $1.90, P=.07)$, and neopterin $(\mathrm{OR}=1.05,95 \% \mathrm{CI} 0.96$ to $1.15, P=.26)$ unsignificant predictors in the SAP group. In the USAP group, $\mathrm{Lp}(\mathrm{a})$ $(\mathrm{OR}=1.29,95 \% \mathrm{CI} 1.12$ to $1.48, P<.0005), \mathrm{TG}(\mathrm{OR}=3.32$, $95 \%$ CI 1.11 to $9.93, P=.03)$, apoA-I $(\mathrm{OR}=0.004,95 \% \mathrm{CI} 0$ to $0.37, P=.02)$, and $\mathrm{CRP}(\mathrm{OR}=1.56,95 \% \mathrm{CI} 1.11$ to 2.18 , $P=.01)$ were significant predictors in comparison with the 
TABLE 7: The results of ROC curves analysis of atherogenic indexes in patients with ischemic heart disease.

\begin{tabular}{|c|c|c|c|c|c|c|c|}
\hline & & AUC & SE & $95 \% \mathrm{CI}$ & Specificity & Sensitivity & Criterion \\
\hline \multirow{6}{*}{ SAP } & apoB/apoA ${ }^{b}$ & 0.717 & 0.062 & $0.594-0.820$ & 78.2 & 64.1 & $>0.93$ \\
\hline & LDL-c/apoB ${ }^{\mathrm{d}}$ & 0.771 & 0.062 & $0.652-0.865$ & 52.7 & 76.9 & $\geq 3.14$ \\
\hline & LDL-c/HDL-c & 0.657 & 0.067 & $0.531-0.769$ & 65.5 & 86.2 & $>3.24$ \\
\hline & Tc/HDL-c ${ }^{\mathrm{d}}$ & 0.737 & 0.060 & $0.615-0.837$ & 63.6 & 74.3 & $>5.08$ \\
\hline & TG/apoB ${ }^{\mathrm{a}, \mathrm{c}}$ & 0.897 & 0.037 & $0.798-0.958$ & 80.0 & 54.3 & $>1.19$ \\
\hline & TG/HDL-c ${ }^{\mathrm{d}, \mathrm{e}}$ & 0.883 & 0.040 & $0.781-0.949$ & 87.0 & 55.9 & $>0.88$ \\
\hline \multirow{6}{*}{ USAP } & apoB/apoA** & 0.896 & 0.034 & $0.807-0.953$ & 56.8 & 82.1 & $>1.08$ \\
\hline & LDL-c/apoB & 0.745 & 0.063 & $0.635-0.836$ & 68.2 & 76.9 & $>3.14$ \\
\hline & LDL-c/HDL-c & 0.840 & 0.043 & $0.741-0.912$ & 53.3 & 93.1 & $>3.40$ \\
\hline & Tc/HDL-c** & 0.873 & 0.038 & $0.780-0.937$ & 73.3 & 71.4 & $>4.97$ \\
\hline & TG/apoB & 0.787 & 0.050 & $0.681-0.871$ & 61.4 & 97.1 & $>1.85$ \\
\hline & TG/HDL-c* & 0.896 & 0.034 & $0.808-0.953$ & 80.0 & 82.4 & $>1.54$ \\
\hline \multirow{6}{*}{ STEMI } & apoB/apo $A^{\mathrm{A}, \mathrm{C}}$ & 0.879 & 0.040 & $0.778-0.945$ & 81.0 & 41.0 & $>0.74$ \\
\hline & LDL-c/apoB & 0.784 & 0.061 & $0.668-0.874$ & 76.2 & 59.0 & $\leq 3.33$ \\
\hline & LDL-c/HDL-c & 0.800 & 0.052 & $0.686-0.887$ & 36.4 & 100.0 & $>3.76$ \\
\hline & Tc/HDL-c ${ }^{B}$ & 0.889 & 0.038 & $0.790-0.952$ & 45.5 & 77.1 & $>5.22$ \\
\hline & TG/apoB & 0.849 & 0.045 & $0.742-0.923$ & 78.6 & 74.3 & $>1.49$ \\
\hline & TG/HDL-c $\mathrm{c}^{\mathrm{A}, \mathrm{D}}$ & 0.912 & 0.034 & $0.819-0.960$ & 88.6 & 55.9 & $>0.88$ \\
\hline
\end{tabular}

${ }^{\mathrm{a}} P<.05$ versus apoB/apoA, LDL-c/apoB and Tc/HDL-c, ${ }^{\mathrm{b}} P<.01$ versus $\mathrm{LDL}-\mathrm{c} / \mathrm{HDL}-\mathrm{c},{ }^{\mathrm{c}} P<.01$ versus LDL-c/HDL-c, ${ }^{\mathrm{d}} P<.001$ versus LDL-c/HDL-c, ${ }^{\mathrm{e}} P<$ .001 versus $\mathrm{Tc} / \mathrm{HDL}-\mathrm{c},{ }^{*} P<.05$ versus $\mathrm{LDL}-\mathrm{c} / \mathrm{apoB}$ and $\mathrm{LDL}-\mathrm{c} / \mathrm{HDL}-\mathrm{c},{ }^{*} P<.05$ versus $\mathrm{LDL}-\mathrm{c} / \mathrm{HDL}-\mathrm{c},{ }^{\mathrm{A}} P<.05$ versus $\mathrm{LDL}-\mathrm{c} / \mathrm{apoB},{ }^{\mathrm{B}} P<.05$ versus $\mathrm{LDL}-$ c/HDL-c, ${ }^{C} P<.01$ versus LDL-c/HDL-c, ${ }^{D} P<.01$ versus TG/apoB.

control group. Finally, in AIM group, only $\mathrm{Lp}(\mathrm{a})(\mathrm{OR}=1.25$, 95\% CI 1.09 to $1.43, P=.001)$ and CRP $(\mathrm{OR}=1.59,95 \%$ CI 1.14 to $2.22, P=.007)$ represented significant predictors comparing to the control group.

\section{Discussion}

The results of this study clearly demonstrated that none of the studied standard and inflammatory markers showed any significant difference between statin-treated and nonstatin-treated patients. However, among lipid markers significantly lower apoA-I values were observed in all statintreated groups, as well as significantly higher apoB ones in STEMI $(+)$ group in comparison with non-statin-treated patients. Since almost all our patients differed only in the statin treatment, observed differences indicate that the statins did not have the same effects on all lipid markers, which made them less sensitive markers.

In this multimarkers study, we showed that $\operatorname{Lp}(\mathrm{a})$ was the marker with the best clinical accuracy, being the marker with the largest AUC and the best sensitivity and specificity among other lipid markers, as well as atherogenic indexes in all patient groups. Of all inflammatory studied markers, hsCRP was found to show significantly higher AUC in comparison with all other inflammatory markers in patients with STEMI. In USAP patients, hsCRP also had the highest AUC although this gained significance only compared to TNF- $\alpha$.

The comparison of hsCRP and Lp(a) ROC curves showed that AUC for $L p(a)$ was higher than for hsCRP in SAP and USAP patients, and a significant difference existed only in USAP patients. In the STEMI group, hsCRP AUC was higher than $\mathrm{Lp}$ (a) AUC, but the difference was not significant. These findings showed that $\operatorname{Lp}(\mathrm{a})$ would be a marker with better clinical accuracy, particularly in patients with USAP what was also confirmed by multinomial logistic regression.

Studies in the past [18] showed that the relationship between coronary artery disease and $\operatorname{Lp}(\mathrm{a})$ was weak and that $\operatorname{Lp}(\mathrm{a})$ may be a marker at only high levels. It could not be used for widespread initial screening because the benefits might be small. Others have observed that the free apo(a) had a better diagnostic test performance in atherosclerotic risk assessment than $\mathrm{Lp}(\mathrm{a})$ testing [19]. Elevated $\mathrm{Lp}(\mathrm{a})$ level is also considered to be the best single marker for the presence of ischemic cerebrovascular disease, and the increased portion of the smaller-molecular-weight apo(a) isoforms in patients and individuals with a sonography score $>0$ points toward an inherited predisposition for this disease [13]. Erbağci et al. [20] showed that for optimal cutoff values for $\mathrm{Lp}(\mathrm{a})$ of 22.6 and $9.8 \mathrm{mg} / \mathrm{dL}$, the diagnostic values of 0.612 and 0.596 in men and women, respectively, with coronary heart disease with or without angiographically demonstrable lesion were found. Our results showed that $\mathrm{Lp}(\mathrm{a})$ had better AUC characteristics and may be more useful than other multimarkers in SAP and USAP patients especially in statin-treated ones.

It was also noted that optimal cutoff levels for hsCRP in women and men were found as 2.1 and $3.0 \mathrm{mg} / \mathrm{L}$ with the diagnostic values of 0.792 and 0.770 , respectively. Contrary to these results, we found much higher diagnostic values of $0.843,0.957$, and 0.888 for $\mathrm{Lp}(\mathrm{a})$ in SAP, USAP, and STEMI patients, as well as higher diagnostic values of 0.859 and 0.946 for hsCRP in USAP and STEMI patients, and the 


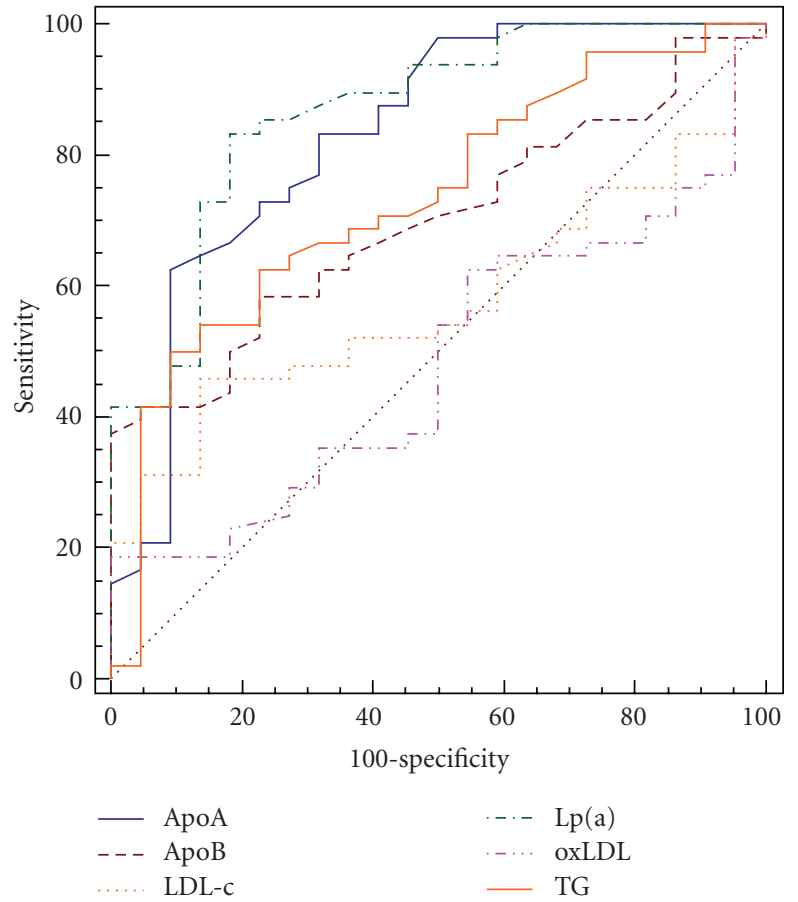

(a)

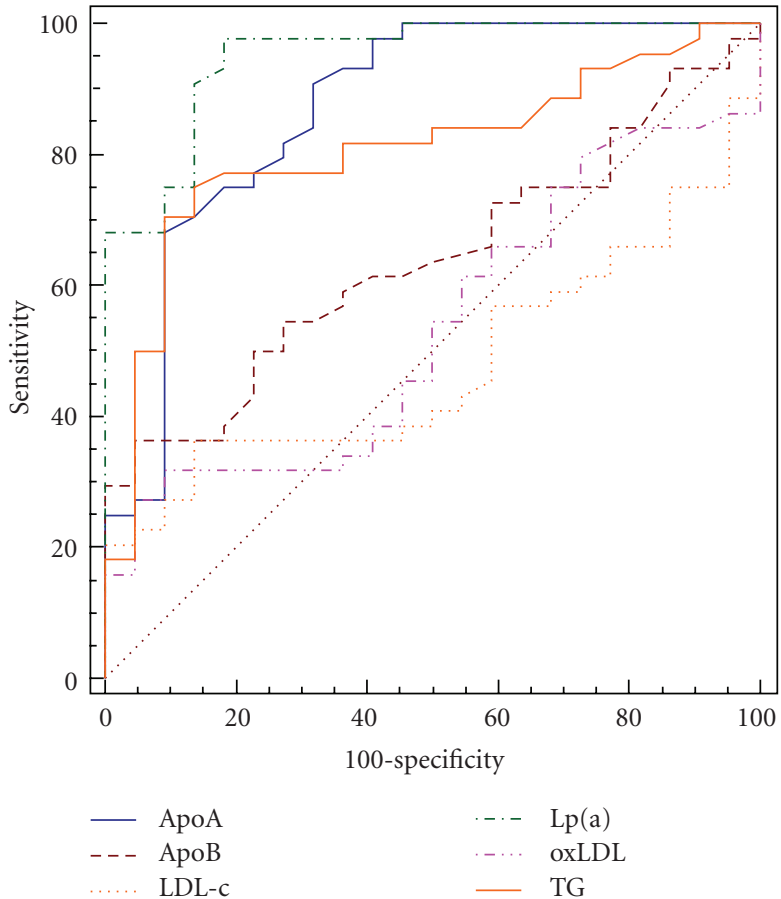

(b)

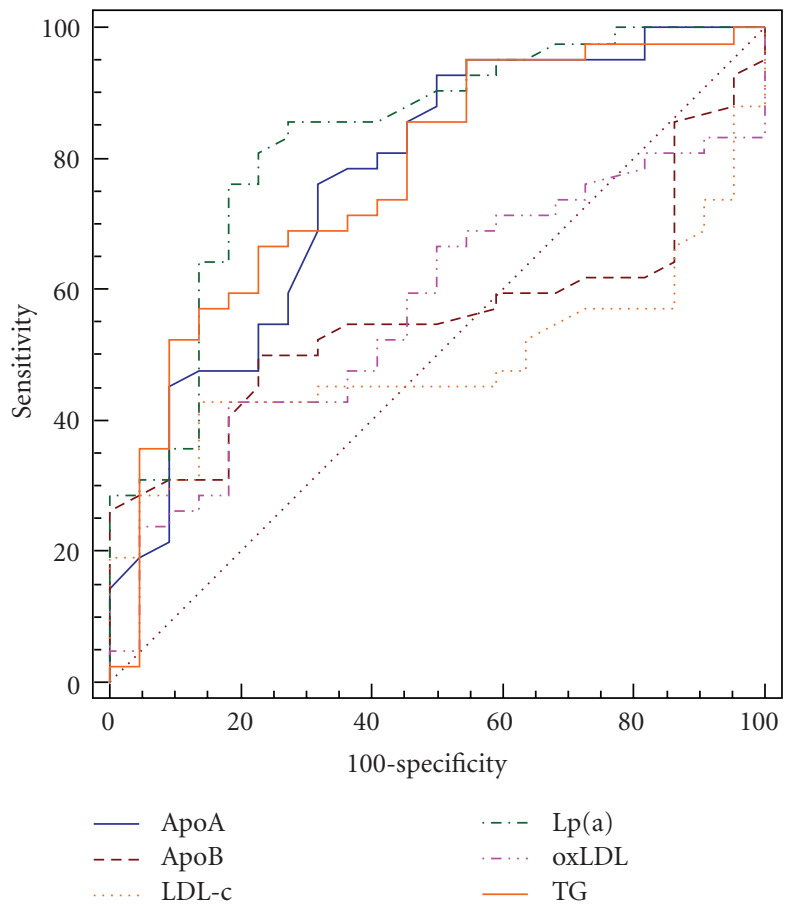

(c)

FIgUre 1: ROC curves of lipid markers: ApoA-I, ApoB, Lp(a), LDL-c, oxLDL, and TG in STEMI patients (a), USAP patients (b), and SAP patients (c).

difference between these two markers was significant in the USAP group. This finding is essential as, taking into account optimal cutoff level as $>8.2$ for $\operatorname{Lp}(\mathrm{a}), \operatorname{Lp}(\mathrm{a})$ is a better independent predictor than CRP in SAP and USAP patients on chronic treatment with statins. The Atherosclerosis Risk in Communities (ARIC) study showed that including some of the additional risk factors in the basic model containing only traditional risk factors might improve predictivity. So, they frequently found that the biggest contributors to the highest increase in AUC, outside the basic model, were albumin, fibrinogen, and $\mathrm{Lp}$ (a) [21]. Bennet et al. [22] noted that baseline $\operatorname{Lp}(\mathrm{a})$ levels had little or no correlation with 


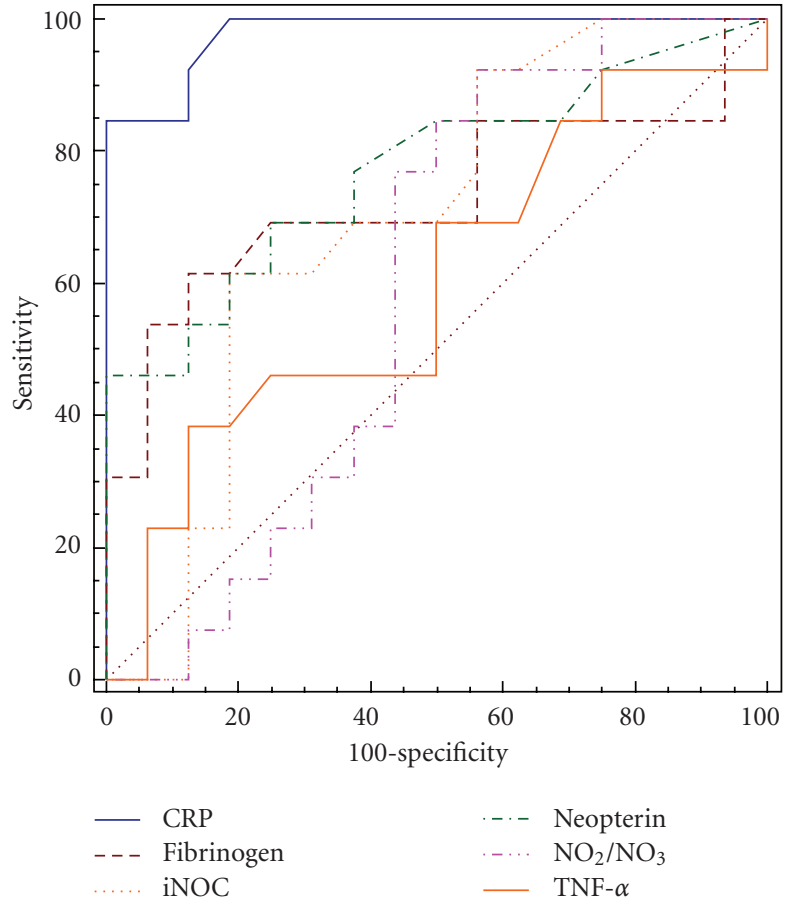

(a)

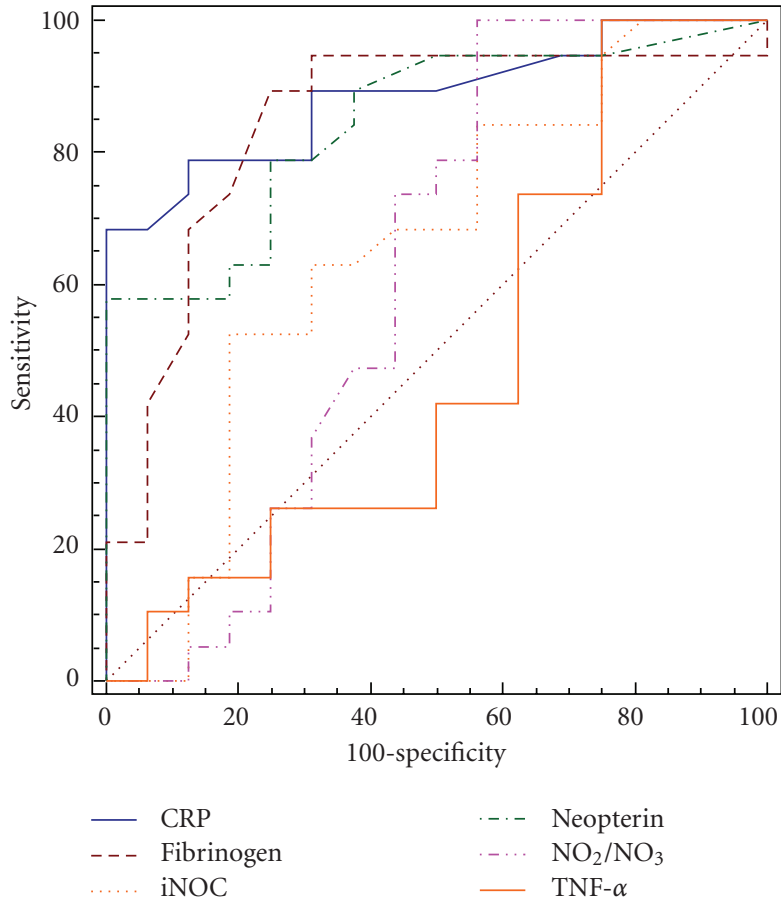

(b)

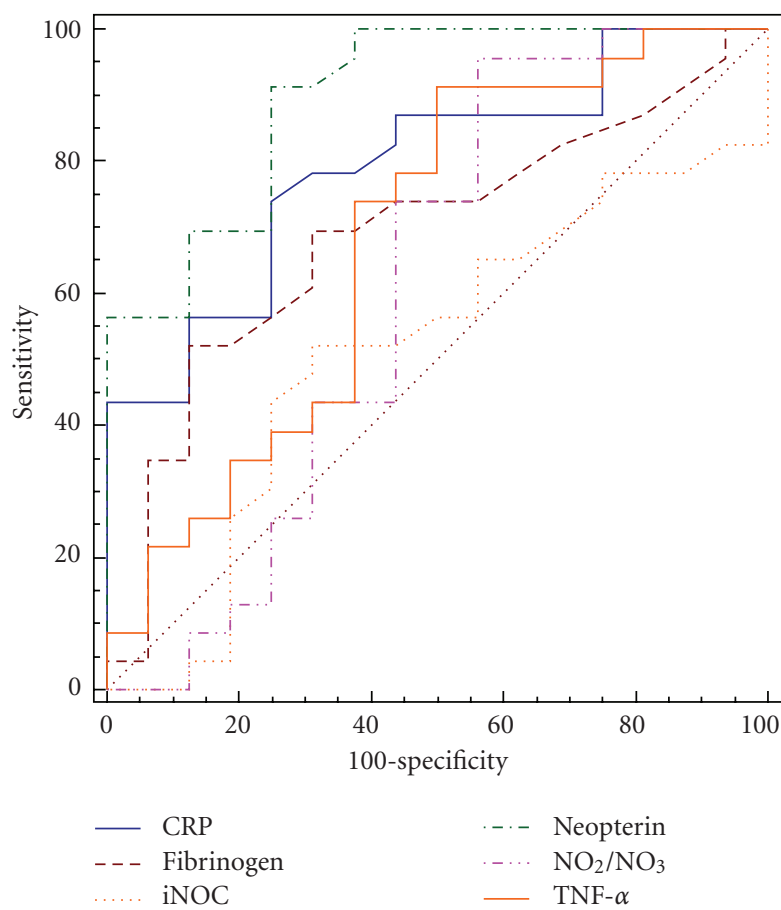

(c)

FIGURE 2: ROC curves of inflammatory markers: CRP, fibrinogen, iNOS, neopterin, $\mathrm{NO}_{2} / \mathrm{NO}_{3}$, and TNF- $\alpha$ in STEMI patients (a), USAP patients (b), and SAP patients (c).

known cardiovascular risk factors including age, sex, total cholesterol level, and blood pressure. They concluded that the levels of $\operatorname{Lp}(\mathrm{a})$ were highly stable within individuals across the time and that there were independent, continuous associations between $\mathrm{Lp}(\mathrm{a})$ levels and a risk of coronary heart disease in a broad range of individuals. It seems that nontraditional risk factors could become more useful in predicting cardiovascular risk since clinical trials of statin therapy have demonstrated that baseline or treated LDLc levels are only weakly associated with a net coronary angiographic change or cardiovascular events [23]. Statins reduce cardiovascular disease events and improve outcomes. 


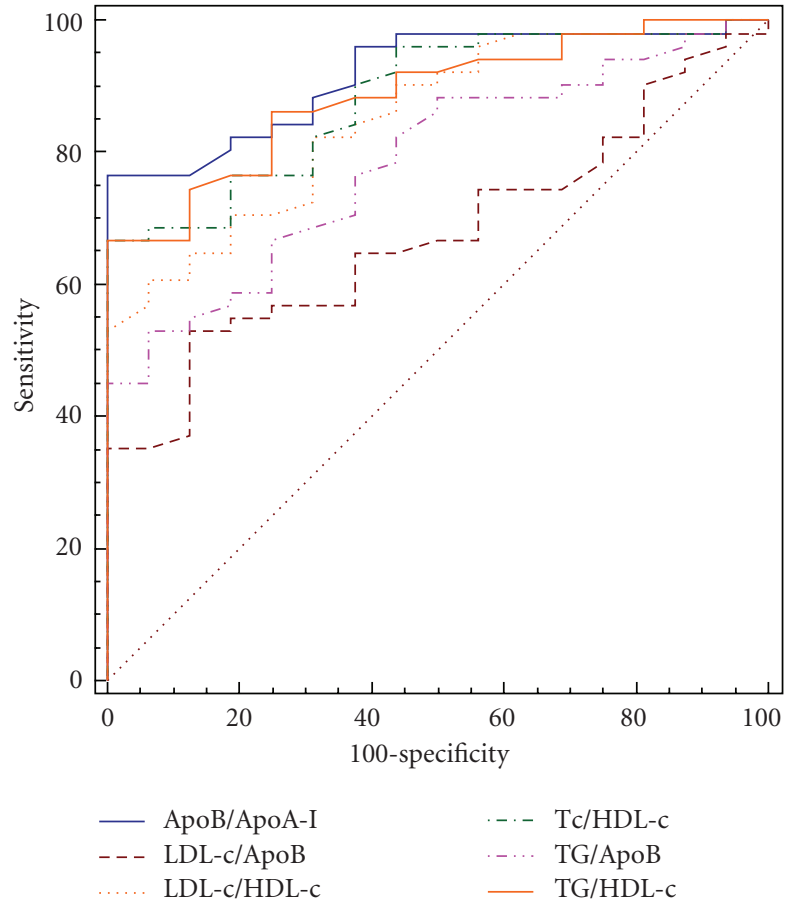

(a)

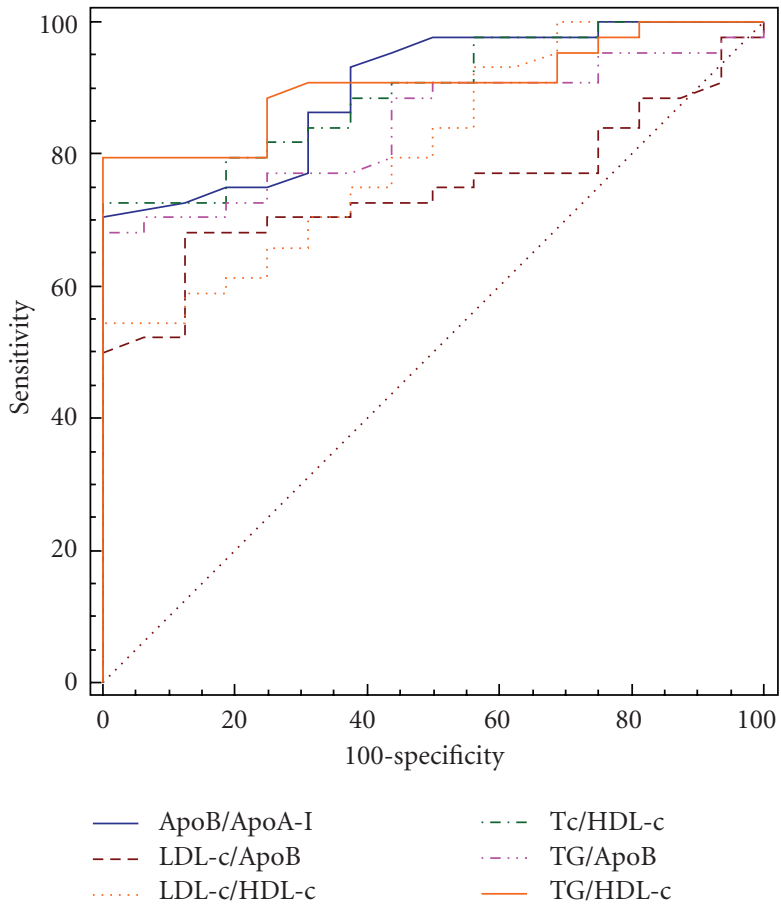

(b)

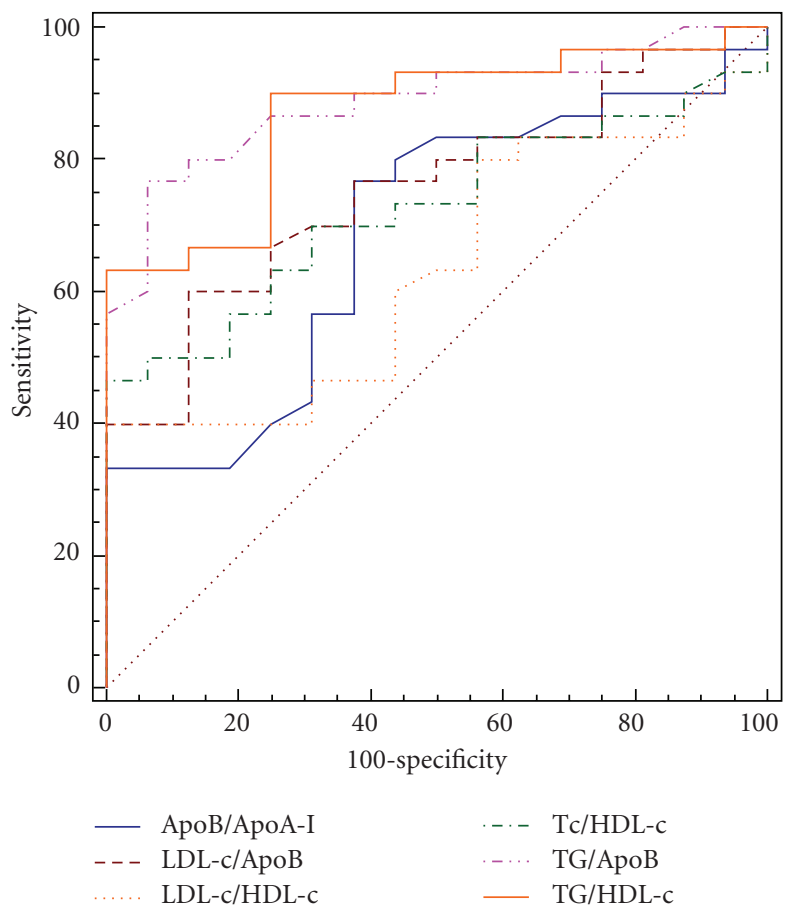

(c)

Figure 3: ROC curves of atherogenic indexes: ApoB/ApoA-I, LDL-c/ApoB, LDL-c/HDL-c, Tc/HDL-c, TG/ApoB, and TG/HDL-c in patients with STEMI (a), USAP (b), and SAP (c).

Large clinical trials indicate that statin-treated individuals have significantly smaller chance of cardiovascular disease, irirespective levels, and that the treatment is particularly effective among patients with high CRP levels. Beside the reduction of lipid and CRP levels [23], statins express additional effects resulting in the improvement of endothelial function, antiinflammatory and antiproliferative response, and the regression of human atherosclerotic lesions [2325]. Contrary to previous findings, in patients with type IIa hypercholesterolemia, both atorvastatin and simvastatin, 
significantly reduced $L p(a)$ levels but not apolipoprotein (a) fragment levels after six-week treatment [26]. In spite of these findings, we did not find any significant difference in $\operatorname{Lp}(\mathrm{a})$ concentration between the statin-treated and nonstatin-treated patients, but we observed significantly lower apoA-I values in all patient groups, and significantly higher apoB in STEMI patients treated with statins.

In a prospective cohort study of more than 15000 healthy women aged 45 years or older, treated with aspirin and vitamin $\mathrm{E}$ and followed up over a 10 -year period, it was noted that non-HDL-c and Tc/HDL-c were as good as, or better than, apolipoprotein fraction in the prediction of future cardiovascular events [27]. While these results support the use of standard lipid measurements, rather than apolipoproteins $\mathrm{A}-\mathrm{I}$ and $\mathrm{B}$ in primary risk detection, in a randomized trial of lovastatin, the levels of apoB and the ratio of apoB/apoAI were better predictors of future cardiovascular events than LDL-c, when participants were receiving the treatment [28]. On this basis, it has been suggested that the measurement of apoB could replace the current lipid status evaluation among patients taking statins [29]. Since our study showed that apoB significantly changed only in STEMI patients, it is not reliable for the evaluation ischemic heart disease patients taking statins. According to our results, it can be $\operatorname{Lp}(\mathrm{a})$.

In conclusion, considering our results, the best marker in assessing ischemic heart disease seemed to be CRP in STEMI, TG/apoB in SAP, and $\mathrm{Lp}(\mathrm{a})$ in USAP patients, respectively.

\section{Statement}

The study was approved by the Clinical Centre Niš Ethical Committee and all the subjects included in study provided written informed consent.

There was no possible conflict of interests considering acception of any funding or support by any organization gaining or loosing from the results of this study. There were no any other conflicting interests at all.

V. B. Djordjević desined the study and wrote the first draft of the manuscript. M. Deljanin-Ilić and L. Popović recruited the patients and wrote the clinical protocol. V. Ćosić and L. Zvezdanović took the blood samples, collected and undertook standard biochemical analyses. I. Stojanović determined nitrite/nitrate concentration in plasma. V. Ćosić, V. B Djordjević, P. Vlahović, and S. Kundalić undertook the determination of TNF- $\alpha$, neopterin, iNOS, and oxLDL. V. B. Djordjević and P. Vlahović undertook the statistical analysis and managed the literature searches and analysis. All authors contributed to and have approved the final paper.

\section{Acknowledgments}

The authors are grateful to Mr. Filip Petrović for the technical assistance in the realization of the paper. This work was financially supported by the Ministry of Science and Technological Development of Serbia.

\section{References}

[1] R. Ross, "Atherosclerosis - an inflammatory disease," New England Journal of Medicine, vol. 340, no. 2, pp. 115-126, 1999.

[2] M. R. Taskinen, "Diabetic dyslipidaemia: from basic research to clinical practice," Diabetologia, vol. 46, no. 6, pp. 733-749, 2003.

[3] K. J. Williams and I. Tabas, "Lipoprotein retention- and clues for atheroma regression," Arteriosclerosis, Thrombosis, and Vascular Biology, vol. 25, no. 8, pp. 1536-1540, 2005.

[4] N. A. Le and M. F. Walter, "The role of hypertriglyceridemia in atherosclerosis," Current Atherosclerosis Reports, vol. 9, no. 2, pp. 110-115, 2007.

[5] J. L. Breslow, "Cardiovascular disease burden increases, NIH funding decreases," Nature Medicine, vol. 3, no. 6, pp. 600-601, 1997.

[6] E. Braunwald, "Shattuck lecture cardiovascular medicine at the turn of the millennium: triumphs, concerns, and opportunities," New England Journal of Medicine, vol. 337, no. 19, pp. 1360-1369, 1997.

[7] C. J. Packard, "LDL subfractions and atherogenicity: an hypothesis from the University of Glasgow," Current Medical Research and Opinion, vol. 13, no. 7, pp. 379-390, 1996.

[8] I. Jialal, D. Stein, D. Balis, S. M. Grundy, B. Adams-Huet, and S. Devaraj, "Effect of hydroxymethyl glutaryl coenzyme a reductase inhibitor therapy on high sensitive C-reactive protein levels," Circulation, vol. 103, no. 15, pp. 1933-1935, 2001.

[9] S. Kinlay, G. G. Schwartz, A. G. Olsson et al., "High-dose atorvastatin enhances the decline in inflammatory markers in patients with acute coronary syndromes in the MIRACL study," Circulation, vol. 108, no. 13, pp. 1560-1566, 2003.

[10] J. B. Muhlestein, J. L. Anderson, B. D. Horne et al., "Early effects of statins in patients with coronary artery disease and high C-reactive protein," American Journal of Cardiology, vol. 94, no. 9, pp. 1107-1112, 2004.

[11] M. A. Albert, E. Danielson, N. Rifai, and P. M. Ridker, "Effect of statin therapy on C-reactive protein levels: the pravastatin inflammation/CRP evaluation (PRINCE): a randomized trial and cohort study," Journal of the American Medical Association, vol. 286, no. 1, pp. 64-70, 2001.

[12] Y. P. Jin, G. M. Qin, S. Z. Zhang, C. J. Li, and G. Xu, "Clinical evaluation of risk factors for coronary heart disease," Zhonghua Yu Fang Yi Xue Za Zhi, vol. 37, no. 2, pp. 98-101, 2003.

[13] G. Jürgens, W. C. Taddei-Peters, P. Költringer et al., "Lipoprotein(a) serum concentration and apolipoprotein(a) phenotype correlate with severity and presence of ischemic cerebrovascular disease," Stroke, vol. 26, no. 10, pp. 1841-1848, 1995.

[14] C. W. Hamm and E. Braunwald, "A classification of unstable angina revisited," Circulation, vol. 102, no. 1, pp. 118-122, 2000.

[15] F. Van de Werf, D. Ardissino, A. Betriu et al., "Task Force on the Management of Acute Myocardial Infarction of the European Society of Cardiology. Management of acute myocardial infarction in patients presenting with ST-segment elevation," European Heart Journal, vol. 24, pp. 28-66, 2003.

[16] V. B. Djordjević, I. Stojanović, V. Ćosić et al., "Serum neopterin, nitric oxide, inducible nitric oxide synthase and tumor necrosis factor- $\alpha$ levels in patients with ischemic heart disease," Clinical Chemistry and Laboratory Medicine, vol. 46, no. 8, pp. 1149-1155, 2008. 
[17] J. A. Navarro-Gonzálvez, C. García-Benayas, and J. Arenas, "Semiautomated measurement of nitrate in biological fluids," Clinical Chemistry, vol. 44, no. 3, pp. 679-681, 1998.

[18] G. A. Hobbs, I. V. Kaplan, and S. S. Levinson, "Mechanized lipoprotein(a) assay as a marker for coronary artery disease illustrates the usefulness of high lipoprotein(a) levels," Clinica Chimica Acta, vol. 274, no. 1, pp. 1-13, 1998.

[19] W. Herrmann, S. Quast, K. Wolter et al., "Determination of free apolipoprotein(a) in serum by immunoassay and its significance for risk assessment in patients with coronary artery disease," Clinical Chemistry and Laboratory Medicine, vol. 37, no. 1, pp. 21-28, 1999.

[20] A. B. Erbağci, M. Tarakçioğlu, M. Aksoy et al., "Diagnostic value of CRP and Lp(a) in coronary heart disease," Acta Cardiologica, vol. 57, no. 3, pp. 197-204, 2002.

[21] L. E. Chambless, A. R. Folsom, A. R. Sharrett et al., "Coronary heart disease risk prediction in the Atherosclerosis Risk in Communities (ARIC) study," Journal of Clinical Epidemiology, vol. 56, no. 9, pp. 880-890, 2003.

[22] A. Bennet, E. Di Angelantonio, S. Erqou et al., "Lipoprotein(a) levels and risk of future coronary heart disease: large-scale prospective data," Archives of Internal Medicine, vol. 168, no. 6, pp. 598-608, 2008.

[23] R. S. Rosenson and C. C. Tangney, "Antiatherothrombotic properties of statins: implications for cardiovascular event reduction," Journal of the American Medical Association, vol. 279, no. 20, pp. 1643-1650, 1998.

[24] J. K. Plenge, T. L. Hernandez, K. M. Weil et al., "Simvastatin lowers C-reactive protein within 14 days: an effect independent of low-density lipoprotein cholesterol reduction," Circulation, vol. 106, no. 12, pp. 1447-1452, 2002.

[25] R. Altman, "Risk factors in coronary atherosclerosis atheroinflammations: the meeting point," Thrombosis Journal, vol. 1, article 4, 2003.

[26] S. Gonbert, S. Malinsky, A. C. Sposito et al., "Atorvastatin lowers lipoprotein(a) but not apolipoprotein(a) fragment levels in hypercholesterolemic subjects at high cardiovascular risk," Atherosclerosis, vol. 164, no. 2, pp. 305-311, 2002.

[27] P. M. Ridker, N. Rifai, N. R. Cook, G. Bradwin, and J. E. Buring, "Non-HDL cholesterol, apolipoproteins A-I and $\mathrm{B}_{100}$, standard lipid measures, lipid ratios, and CRP as risk factors for cardiovascular disease in women," Journal of the American Medical Association, vol. 294, no. 3, pp. 326-333, 2005.

[28] A. M. Gotto, E. Whitney, E. A. Stein et al., "Relation between baseline and on-treatment lipid parameters and first acute major coronary events in the Air Force/Texas Coronary Atherosclerosis Prevention Study (AFCAPS/TexCAPS)," Circulation, vol. 101, no. 5, pp. 477-484, 2000.

[29] S. Miremadi, A. Sniderman, and J. Frohlich, "Can measurement of serum apolipoprotein B replace the lipid profile monitoring of patients with lipoprotein disorders?" Clinical Chemistry, vol. 48, no. 3, pp. 484-488, 2002. 


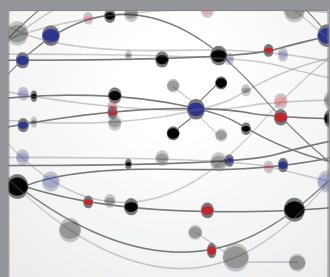

The Scientific World Journal
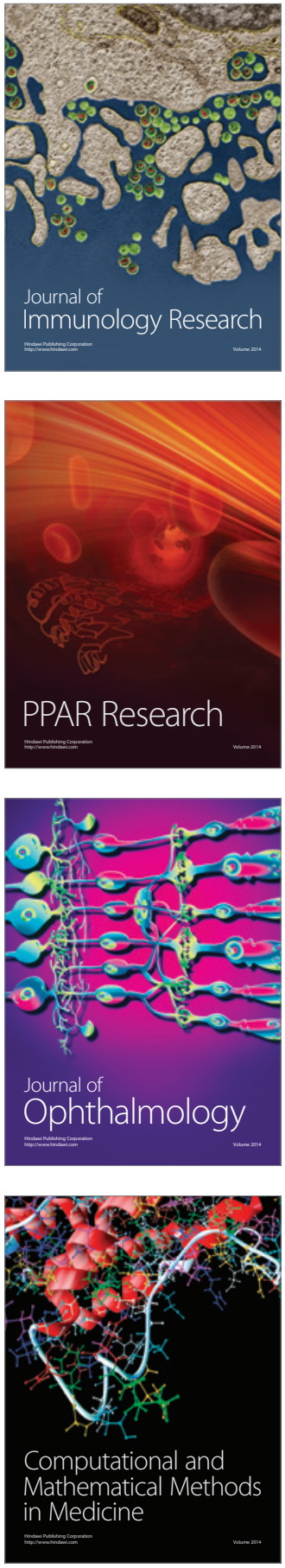

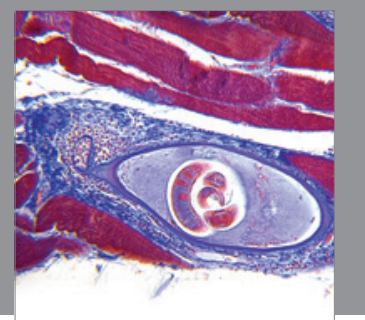

Gastroenterology

Research and Practice
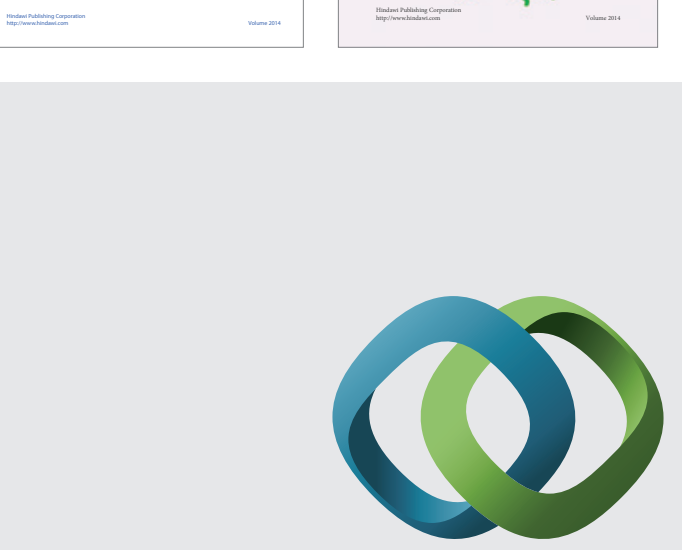

\section{Hindawi}

Submit your manuscripts at

http://www.hindawi.com
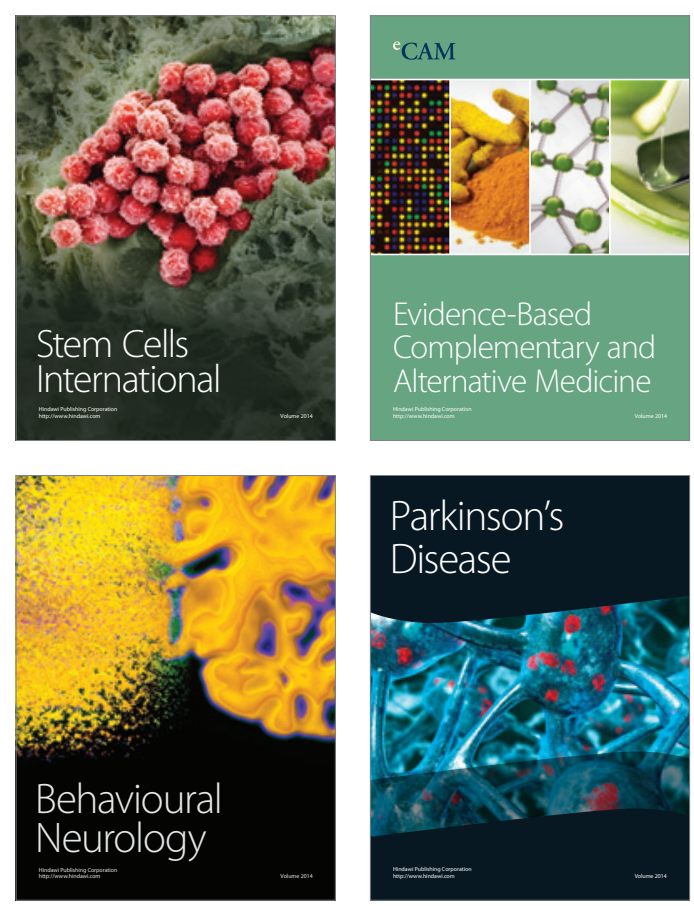

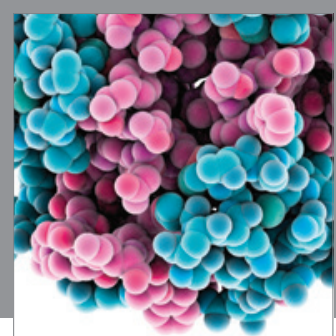

Journal of
Diabetes Research

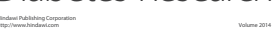

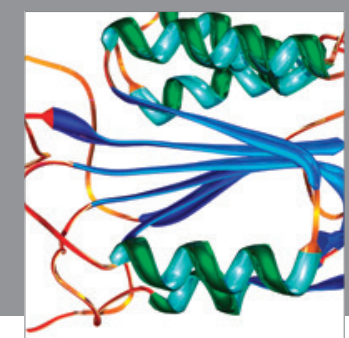

Disease Markers
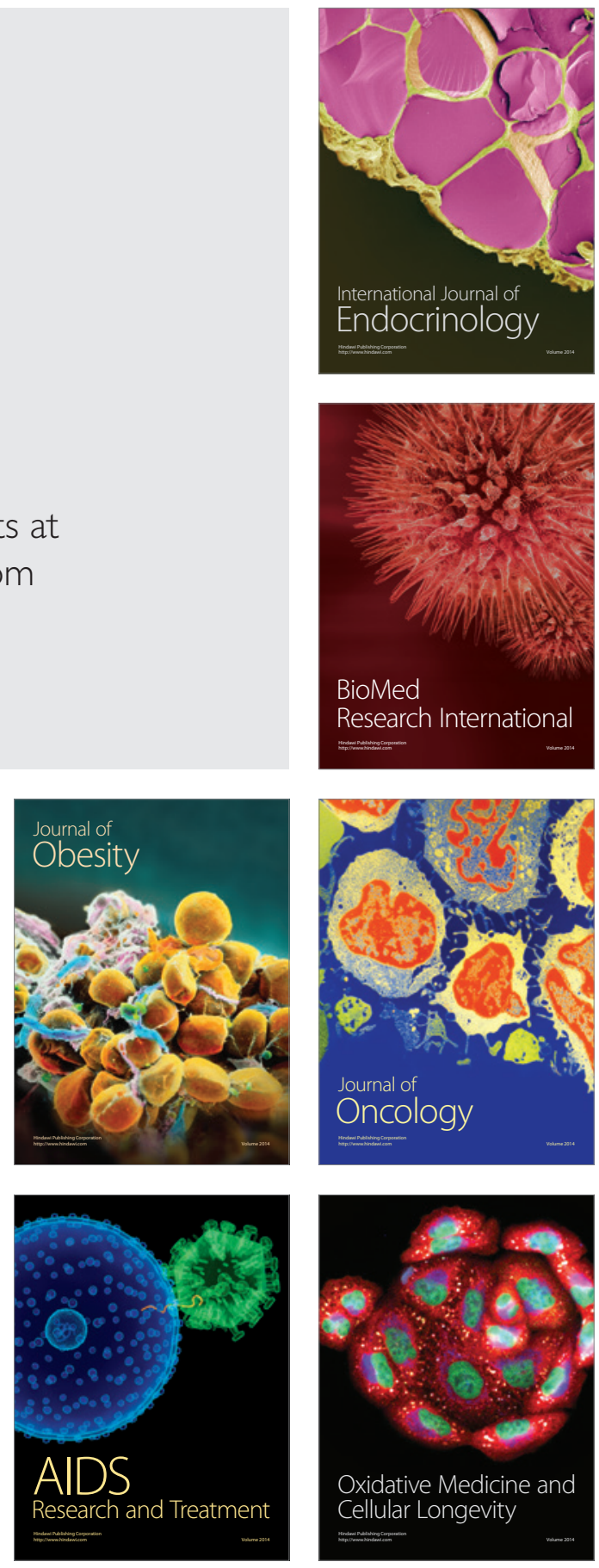